\title{
One-Year Results of the BeweegKuur Lifestyle Intervention Implemented in Dutch Primary Healthcare Settings
}

\author{
Bianca A. M. Schutte, ${ }^{1,2}$ Annemien Haveman-Nies, ${ }^{1}$ and Liesbeth Preller ${ }^{2}$ \\ ${ }^{1}$ Division of Human Nutrition, Wageningen University, Bomenweg 4, 6703 HD Wageningen, Netherlands \\ ${ }^{2}$ Netherlands Institute for Sport and Physical Activity, Horapark 4, 6717 LZ Ede, Netherlands \\ Correspondence should be addressed to Bianca A. M. Schutte; a.m.schutte@lumc.nl
}

Received 20 November 2014; Revised 30 March 2015; Accepted 12 May 2015

Academic Editor: Subhash Pokhrel

Copyright (C) 2015 Bianca A. M. Schutte et al. This is an open access article distributed under the Creative Commons Attribution License, which permits unrestricted use, distribution, and reproduction in any medium, provided the original work is properly cited.

\begin{abstract}
Background. Lifestyle interventions focusing on healthy diet and physical activity (PA) are effective in reducing health risks in controlled research settings. The aim of this study was to investigate the one-year results of the BeweegKuur lifestyle intervention implemented nationwide in Netherlands for people with a weight-related health risk. Materials and Methods. Data were requested from all 160 locations participating in the BeweegKuur. In a one group pretest/posttest study, one-year changes in health outcome variables and time spent on physical activity were tested with dependent $t$-tests. Associations between one-year changes in weight and waist circumference and sociodemographic factors and uptake of the program were analysed with ANOVA. Results. Data for 517 participants from 47 locations were available for analysis. One year after the intervention, weight reduced by $2.9 \mathrm{~kg}(95 \% \mathrm{CI}$ $-3.3 ;,-2.5)$, waist circumference by $4.3 \mathrm{~cm}(-4.9 ;-3.7)$, and blood glucose by $0.5 \mathrm{mmol} / \mathrm{L}(-0.8 ;-0.3)$. Physical activity increased significantly. Higher uptake of the program was associated with a larger decrease in waist circumference. Conclusion. The results of the study suggest that lifestyle interventions implemented in real-life primary healthcare settings with tailor-made supervision can contribute meaningfully to primary prevention.
\end{abstract}

\section{Introduction}

Worldwide, age-standardized prevalence of obesity in adults almost doubled between 1980 and 2008 [1]. Obesity is a significant risk factor for increased morbidity and mortality, most importantly for cardiovascular disease and type 2 diabetes mellitus $[2,3]$. As inactivity and an unhealthy diet are very strong determinants of obesity, cardiovascular disease, and type 2 diabetes, much of the disease burden can be prevented or at least be postponed [4-6]. Lifestyle interventions combining a focus on sustainable change in diet, physical activity (PA), and behavioural change demonstrate promising results in reducing the risk of obesity and type 2 diabetes [79]. However, most lifestyle interventions aimed at reducing diabetes and cardiovascular risks are developed and tested in controlled research settings. They are usually intensive, highly standardized, and delivered by specially educated staff using strict protocols [10]. Different studies have been performed to determine whether results obtained in research settings could be replicated in real-life primary healthcare [11-15]. These interventions are implemented by regular primary healthcare professionals (general practitioner, practice nurse, dietician, and/or physiotherapist), and participants are supervised for a shorter intervention period than in research, of about one year. Overall, effects of these real-life interventions yield smaller effect sizes for health outcome variables compared to interventions in controlled research settings, or no effects are found [16].

Within a nationwide implementation project commissioned by the Dutch Ministry of Health, Welfare, and Sport, the BeweegKuur was implemented in local, real-life primary healthcare settings spread over Netherlands [17]. The objective of this study is to evaluate the effect of this lifestyle intervention on weight, waist circumference, and PA. In addition, the association between change in weight and waist circumference and level of uptake of the program and participants' sociodemographic characteristics is studied. 


\section{Materials and Methods}

2.1. The BeweegKuur Lifestyle Intervention. The pilot BeweegKuur project was started in 2007 because the former Dutch Minister of Health wanted to implement a lifestyle intervention nationwide, accessible, and reimbursed by health insurance for the whole target group. Together with national stakeholders, an intervention was designed based on existing interventions and known effective elements. The intervention took place in the primary healthcare setting. The aim of the project was to optimize nationwide implementation [17]. This was accompanied by process evaluations [18, 19]. On the basis of these results, individual locations were allowed to adjust the BeweegKuur to a certain extent to the local situation. Also, these locations were allowed to use their own quality-assured procedures for testing and measuring health parameters. Locations applied voluntarily to join, resulting in 160 participating locations spread over Netherlands in 2011. Every location was allowed to include a maximum of 40 participants per year. Local healthcare professionals were supported by one of 20 regional primary care support structures, which in turn were instructed by the Netherlands Institute for Sport and Physical Activity (NISB). All professionals supporting participants received training on the BeweegKuur; lifestyle advisors (LSAs) and physiotherapists received a three-day course and the dieticians a one-day course. BeweegKuur protocols and other guidelines were available for all healthcare professionals.

The aim of the BeweegKuur intervention in itself was to achieve health benefits through increased PA and improved dietary behaviour. The BeweegKuur's starting point was the general practitioner's (GP) practice. The GP referred patients with a weight-related health risk (see Section 2.2 for inclusion criteria) to an intake session with an LSA, commonly a practice nurse. Shortly afterwards, the exact time depending on the location, a physiotherapist performed an endurance test. The LSA used this test to assign the participant to exercise programs 1,2 , or 3 , varying in intensity of guidance by the LSA and physiotherapist (Table 1). Throughout the year, participants had around seven tailor-made coaching and supervision sessions with the LSA based on principles of motivational interviewing [20] and the self-determination theory [21]. In addition, all participants were referred to a dietician. Participants could start with the intervention whenever it suited them, in consultation with the LSA, but the duration of the intervention was one year for all participants. To increase PA and to contribute to sustainable changes in PA, participants were coached to increase PA in daily life and referred to local exercise facilities during the intervention. Again, participants had flexibility about when they could start at these facilities; the timing depended on the ability of the participant to exercise individually. The timing and type of all activities were highly tailored to the participant and designed in close consultation with the participant and the physiotherapist. The number of consultations with the LSA, dietician, and physiotherapist was higher in the beginning of the intervention and decreased gradually during the year.
TABLE 1: Number of consultations with the different healthcare professionals per exercise program.

\begin{tabular}{lccc}
\hline & $\begin{array}{c}\text { Program 1 } \\
\text { Independent } \\
\text { exercise } \\
\text { program }^{1}\end{array}$ & $\begin{array}{c}\text { Program 2 } \\
\text { Start-up } \\
\text { program }\end{array}$ & $\begin{array}{c}\text { Program 3 } \\
\text { Supervised } \\
\text { exercise } \\
\text { program }^{1}\end{array}$ \\
\hline $\begin{array}{l}\text { Lifestyle } \\
\text { advisor }\end{array}$ & 8 & 7 & 7 \\
$\begin{array}{l}\text { Physiotherapist } \\
\text { Individual }\end{array}$ & 0 & 5 & $24-51^{*}$ \\
$\begin{array}{l}\text { sessions } \\
\text { dietician }\end{array}$ & 4 & 4 & 4 \\
$\begin{array}{l}\text { Group sessions } \\
\text { dietician }\end{array}$ & 7 & 7 & 7 \\
\hline $\begin{array}{l}1 \\
\text { Number of consultations was higher at the beginning of the intervention } \\
\text { and decreased gradually during the year. } \\
{ }^{*} 2-3 \text { sessions a week for 3-4 months. }\end{array}$ &
\end{tabular}

2.2. Study Design and Participants. In this study, a one group pretest/posttest study design was used. Data were collected per location by the LSA and entered in a standardized registration file, administered by NISB. In autumn 2011, these registration files were requested for this study from the LSAs in all 160 locations. The majority of participants started with the intervention in 2009 and 2010.

The GP was responsible for screening for contraindications, based on current medical guidelines and standards in the Netherlands, and decided whether a person could participate in the BeweegKuur. Prospective participants had to meet certain inclusion criteria. They were included if they

(i) were motivated to change behaviour;

(ii) did not meet the Dutch Standard for Healthy Exercise (at least 30 minutes of moderate physical activity on at least five days a week);

(iii) had a BMI between 25 and 30 in combination with a waist circumference $\geq 88 \mathrm{~cm}$ for women and $\geq 102 \mathrm{~cm}$ for men and/or comorbidity;

(iv) had a BMI between 30 and 35, regardless of waist circumference and comorbidity;

(v) had a BMI between 35 and 40, regardless of waist circumference but without comorbidity.

Comorbidity included hypertension, dyslipidaemia, diabetes mellitus, cardiovascular disease, osteoarthritis, and sleep apnoea.

2.3. Measurements. Measurements were taken during sessions with the LSA and included weight, BMI, waist circumference, blood glucose, and blood pressure. Data from the first and last sessions were used for the analyses. Anthropometric and blood pressure measurements were conducted according to standardized procedures in the GP practice. For blood glucose, LSAs registered whether blood glucose was measured in a fasting state or not, according to local procedures. Only fasting-state measurements were used for analysis. 
Physical activity was self-reported using the standardized short version of the validated SQUASH questionnaire [22]. Activities performed at work, during household activities, leisure time, and commuting were classified into the categories light to moderate and vigorous on the basis of their intensity. Sociodemographic factors like age, gender, smoking behaviour, and educational level were recorded by the LSA using standardized questionnaires. To assess uptake of the program, the number of sessions with the dietician and the LSA and attendance at the group education lessons with the dietician were recorded by the LSA.

2.4. Statistical Analysis. Data analyses were conducted using SPSS for Windows (version 18). One-year changes in weight, BMI, waist circumference, blood glucose, blood pressure, and physical activity were normally distributed for the overall population and the different subgroups. One-year changes were tested with a paired samples' $t$-test. A $p$ value of $<0.05$ was considered to be statistically significant. All tests were two-sided. ANOVA was conducted to test whether sociodemographic factors, uptake of the program, and change in physical activity were related to changes in weight and waist circumference, followed by the Games-Howell post hoc test $(p<0.05)[23]$.

\section{Results}

The LSAs from 81 of the 160 locations sent back the registration files. Complete data for 517 participants from 47 locations (mean $=11$ persons/location) were available for analysis; participants in the other locations had not yet finished the intervention at the time of data retrieval.

The background characteristics of participants are described in Table 2. Mean age was 58 years; most participants were between 50 and 70 years old. The majority of participants were female (59.2\%). Most participants had low (39.5\%) or intermediate $(44.4 \%)$ levels of education. Compared to the Dutch population of the same age, participants were less educated and more often married (Supplementary Table 1 in Supplementary Material available online at http://dx.doi.org/10.1155/2015/484823).

One year after the start of the intervention, weight and waist circumference had significantly reduced by $2.9 \mathrm{~kg}$ (3.0\% of baseline) and $4.3 \mathrm{~cm}$ (3.8\%) (Table 3). Other health parameters showed the same trend. Light to moderate PA and vigorous PA increased by 2.1 (15\%) and 1.7 (40\%) hours a week, respectively. Males and females differed in the anthropometric outcomes and PA at baseline, but the effects on weight and waist circumference were similar for men and women (Supplementary Table 2).

Younger participants lost on average more weight than older participants (Table 4). Larger reductions in weight and waist circumference were seen in participants in the highest BMI category. Generally, larger changes in waist circumference were associated with a higher uptake of the program: waist circumference decreased more in participants with more supervision from the physiotherapist and six or more sessions with the LSA.
TABLE 2: Sociodemographic characteristics of the BeweegKuur intervention participants.

\begin{tabular}{lcc}
\hline & $N$ & Study population \\
\hline $\begin{array}{l}\text { Age (years), mean (SD) } \\
\text { Sex (\%) }\end{array}$ & 511 & $58.2(10.9)$ \\
$\quad$ Male & 210 & 40.8 \\
$\quad$ Female & 305 & 59.2 \\
Civil status (\%) & & \\
$\quad$ Married & 368 & 73.5 \\
$\quad$ Living together & 29 & 5.8 \\
$\quad$ Divorced & 24 & 4.8 \\
$\quad$ Widow/widower & 25 & 5.0 \\
$\quad$ Single & 55 & 11.0 \\
Education (\%) & & \\
$\quad$ Lower education & 123 & 39.5 \\
Intermediate education & 138 & 44.4 \\
$\quad$ High education & 50 & 16.1 \\
Smoking behaviour (\%) & & \\
$\quad$ Smoker & 28 & 13.6 \\
$\quad$ Nonsmoker & 178 & 86.4 \\
\hline
\end{tabular}

Total number of participants is not similar for all sociodemographic characteristics as complete data are not available for all participants.

\section{Discussion}

This study has shown that participation in the real-life lifestyle intervention BeweegKuur was positively related to participants reducing weight, waist circumference, blood pressure, and blood glucose and increasing their physical activity. The largest changes in waist circumference were observed in participants with higher uptake of the program.

In this study, weight and waist circumference reduced by $2.9 \mathrm{~kg}$ and $4.3 \mathrm{~cm}$, respectively, after one year. These effects were stronger than that found in a previous effect evaluation of an earlier and less developed version of the BeweegKuur carried out in a few locations [24]. The larger effects in this study may be caused by an increase in the professional development of LSAs, physiotherapists, and dieticians as well as the improved attention given to PA outside the intervention. Also, in comparison with other lifestyle interventions implemented in practice, that is, Australian Greater Green Triangle (GGT) Diabetes Prevention Project [12], the Dutch APHRODITE study [15], the Finnish GOAL Intervention Study [11], and the Finnish National Diabetes Prevention Program (FIN-D2D) [14], the BeweegKuur appeared to be more effective. In these interventions, weight loss was on average between 0.5 and $2.5 \mathrm{~kg}$ and waist circumference decreased from 0.4 to $4.2 \mathrm{~cm}$ after 1 to 1.5 years.

In the BeweegKuur, participants were referred to the most appropriate exercise program, depending on the level of weight-related health risk. Participants followed sessions with the physiotherapist, and the LSA designed a tailor-made program and provided coaching and supervision, according to the person's needs. Additionally, participants were referred to local sport facilities with personalized programs adjusted to their physical and mental capabilities. In none of the 
TABLE 3: Baseline measurements and changes in anthropometric outcomes and physical activity after one year.

\begin{tabular}{lccc}
\hline & $N$ & Baseline $^{1}$ & Change $^{1}$ \\
\hline Weight $(\mathrm{kg})$ & 515 & $95.6(94.0 ; 97.2)$ & $-2.9(-3.3 ;-2.5)^{* * *}$ \\
BMI $\left(\mathrm{kg} / \mathrm{m}^{2}\right)$ & 515 & $33.0(32.5 ; 33.5)$ & $-1.0(-1.2 ;-0.9)^{* * *}$ \\
Waist circumference $(\mathrm{cm})$ & 395 & $110.4(109.1 ; 111.7)$ & $-4.3(-4.9 ;-3.7)^{* * *}$ \\
Blood glucose $(\mathrm{mmol} / \mathrm{L})$ & 257 & $7.5(7.2 ; 7.7)$ & $-0.5(-0.7 ;-0.3)^{* * *}$ \\
Systolic blood pressure $(\mathrm{mmHg})$ & 434 & $138.8(137.3 ; 140.2)$ & $-3.3(-4.8 ;-1.9)^{* * *}$ \\
Diastolic blood pressure $(\mathrm{mmHg})$ & 432 & $82.4(81.6 ; 83.3)$ & $-2.6(-3.4 ;-1.7)^{* * *}$ \\
Light to moderate physical activity (hours/week) & 395 & $13.6(12.3 ; 14.9)$ & $2.1(1.0 ; 3.2)^{* * *}$ \\
Vigorous physical activity (hours/week) & 251 & $4.3(3.5 ; 5.0)$ & $1.7(0.8 ; 2.5)^{* * *}$ \\
\hline
\end{tabular}

${ }^{1}$ Data are mean (95\% confidence interval). ${ }^{* * *}$ Statistical significant difference, paired sample $t$-test $(p<0.001)$.

other intervention studies participants received similar tailormade supervision to increase their PA $[11,12,14,15,25]$. The increase in PA was also larger in the BeweegKuur compared with other intervention studies. In the BeweegKuur, light to moderate and vigorous PA were increased by 2.1 and 1.7 hours a week, respectively. The Hoorn Prevention Study found a decrease in PA after one year [13] and the APHRODITE study found an increase in PA after half a year and a decrease after 1.5 and 2.5 years $[15,26]$. The considerable effect of the BeweegKuur on PA may be due, at least partly, to the strong focus on optimal implementation, based on the results of extensive formative evaluation in relation to the nationwide implementation project $[18,19]$.

In the BeweegKuur, participants had individual sessions with the LSA, but most sessions with the dietician and the physiotherapist were in groups. Alongside the benefits of individual coaching, group counselling can promote group cohesion, generally having a beneficial effect on behaviour change [17, 19, 27-29]. In other interventions implemented in practice, most sessions with healthcare professionals were individual, or the number of group sessions was minimal [14, 25]. Moreover, the BeweegKuur was an extensive program, and uptake of the program was high in comparison with other interventions. Fifty-five percent of BeweegKuur participants visited the LSA six times or more. In the FIN-D2D program, only $29 \%$ of the participants had three or more sessions in usual primary healthcare [14], and $43 \%$ and $57 \%$ of the participants attended a maximum of six counselling sessions in the GGT Diabetes Prevention Project and the GOAL intervention, respectively $[11,12]$. We found stronger effects on waist circumference in persons who had more consultations with primary healthcare professionals and attended group sessions.

Initially, the BeweegKuur was designed as an implementation project aimed at optimizing local and nationwide implementation of lifestyle interventions and not primarily as a research project [17]. Consequently, the BeweegKuur was well embedded in local-practice working standards, but the structure of the project led to several drawbacks for this study. No control group could be included and the response rate was relatively low, thereby limiting adjustment for potential confounders.

The design of the whole project did not include very strict quality control procedures for data collection. Individual
LSAs were responsible for data entering. This might have led to potential information bias, with structural overestimation of the results of the intervention. Differences in measurement methods could also have led to bias but are unlikely to contribute to overestimation of associations. All measurements were carried out with instruments and tests available in professional general practices. These instruments meet professional quality standards, and therefore we believe that the effect on measured differences is within the range of total variation of these parameters.

The study locations included in the analysis were spread across the country, representing the Dutch target group. The study population might be a selective group, as data for persons who did not complete the BeweegKuur were not collected. In Vermunt et al.s study [30] on the effectiveness of the Dutch APHRODITE study, a lifestyle intervention in primary healthcare, it was observed that dropouts had similar clinical outcomes (body weight, blood glucose values) on baseline as completers.

Notwithstanding these methodological limitations, this study can contribute to a growing understanding of an effective implementation methodology regarding lifestyle interventions in real-life primary care settings, as knowledge on this essential step is limited. This study gives an indication that the essential elements of the intervention seem to be a number of sessions with the healthcare professionals, a combination of individual and group sessions, tailor-made supervision and counselling, and referral to local sport facilities with personalized programs. The latter element is described in detail by Elsman et al. [29].

\section{Conclusion}

One-year results of the BeweegKuur lifestyle intervention demonstrated positive results on physical activity and anthropometric outcomes. Due to limitations of the study design, it cannot be ruled out that reported outcomes overestimate the results that can be achieved in the entire population. However, the effect evaluation indicates that a well-implemented intervention, combining individual and group sessions and tailor-made supervision by local healthcare professionals, can result in substantial lifestyle and health changes in persons who fully participate. 
TABLE 4: Changes in weight and waist circumference after one year, comparison between different subgroups.

\begin{tabular}{|c|c|c|c|c|}
\hline & \multicolumn{2}{|c|}{ Weight change } & \multicolumn{2}{|c|}{ Waist circumference change } \\
\hline & $N$ & $(\mathrm{~kg})^{1,2}$ & $N$ & $(\mathrm{~cm})^{1,2}$ \\
\hline \multicolumn{5}{|l|}{ Sociodemographic factors } \\
\hline \multicolumn{5}{|l|}{ Sex } \\
\hline Male & 210 & $-3.1(-3.7 ;-2.5)$ & 172 & $-4.5(-5.3 ;-3.6)$ \\
\hline Female & 305 & $-2.8(-3.4 ;-2.2)$ & 223 & $-4.2(-5.1 ;-3.3)$ \\
\hline \multicolumn{5}{|l|}{ Age (years) } \\
\hline$<55$ & 188 & $-3.8(-4.7 ;-3.0)^{* * a}$ & 137 & $-4.1(-5.2 ;-3.1)$ \\
\hline $55-65$ & 187 & $-2.6(-3.3 ;-1.8)$ & 157 & $-4.4(-5.5 ;-3.4)$ \\
\hline$>65$ & 136 & $-2.2(2.7 ;-1.6)^{\mathrm{a}}$ & 98 & $-4.4(-5.7 ;-3.1)$ \\
\hline \multicolumn{5}{|l|}{ BMI at baseline $\left(\mathrm{kg} / \mathrm{m}^{2}\right)$} \\
\hline$<30$ & 174 & $-2.1(-2.6 ;-1.5)^{* * * a}$ & 133 & $-3.9(-4.8 ;-2.9)^{*}$ \\
\hline $30-35$ & 199 & $-2.6(-3.2 ;-1.9)^{\mathrm{b}}$ & 154 & $-3.7(-4.6 ;-2.7)^{\mathrm{a}}$ \\
\hline$>35$ & 144 & $-4.4(-5.4 ;-3.4)^{\mathrm{a}, \mathrm{b}}$ & 108 & $-5.8(-7.3 ;-4.3)^{\mathrm{a}}$ \\
\hline \multicolumn{5}{|l|}{ Education } \\
\hline Lower education & 123 & $-3.1(-4.0 ;-2.3)$ & 105 & $-5.2(-6.7 ;-3.8)$ \\
\hline Intermediate Education & 138 & $-3.1(-3.9 ;-2.3)$ & 119 & $-4.7(-5.9 ;-3.5)$ \\
\hline High education & 50 & $-3.2(-4.5 ;-1.9)$ & 39 & $-4.1(-5.8 ;-2.4)$ \\
\hline \multicolumn{5}{|l|}{ Uptake of the program } \\
\hline \multicolumn{5}{|l|}{ Exercise program } \\
\hline (1) independent program & 158 & $-2.6(-3.4 ;-1.8)$ & 123 & $-3.1(-4.1 ;-2.2)^{* \mathrm{a}}$ \\
\hline (2) start-up program & 163 & $-3.3(-4.0 ;-2.5)$ & 124 & $-4.7(-5.8 ;-3.6)$ \\
\hline (3) supervised program & 166 & $-2.9(-3.7 ;-2.1)$ & 132 & $-5.1(-6.4 ;-3.8)^{\mathrm{a}}$ \\
\hline \multicolumn{5}{|c|}{ Number of individual sessions with dietician } \\
\hline $1-4$ & 239 & $-2.6(-3.1 ;-2.1)$ & 196 & $-4.1(-5.0 ;-3.3)$ \\
\hline 4 or more & 159 & $-3.5(-4.4 ;-2.5)$ & 126 & $-5.1(-6.4 ;-3.8)$ \\
\hline \multicolumn{5}{|c|}{ Attendance group education sessions } \\
\hline no & 75 & $-1.7(-2.8 ;-0.7)$ & 47 & $-1.4(-2.8 ; 0.1)^{*}$ \\
\hline yes & 150 & $-2.3(-3.1 ;-1.6)$ & 127 & $-3.5(-4.7 ;-2.3)$ \\
\hline \multicolumn{5}{|l|}{ Number of sessions with LSA } \\
\hline $1-3$ & 205 & $-2.4(-3.0 ;-1.8)$ & 156 & $-3.3(-4.3 ;-2.4)^{*}$ \\
\hline 4 or more & 253 & $-3.1(-3.8 ;-2.5)$ & 201 & $-4.8(-5.7 ;-3.9)$ \\
\hline \multicolumn{5}{|l|}{ Changes in physical activity } \\
\hline \multicolumn{5}{|c|}{ Change light and moderate physical activity (hours) } \\
\hline$<0$ & 137 & $-2.3(-3.2 ;-1.5)$ & 102 & $-3.9(-4.9 ;-2.8)$ \\
\hline $0-3.5$ & 116 & $-2.8(-3.7 ;-1.9)$ & 89 & $-4.2(-5.8 ;-2.7)$ \\
\hline$>3.5$ & 123 & $-3.2(-4.1 ;-2.4)$ & 113 & $-5.1(-6.3 ;-3.9)$ \\
\hline \multicolumn{5}{|c|}{ Change vigorous physical activity (hours) } \\
\hline$<0$ & 82 & $-2.4(-3.7 ;-1.2)$ & 58 & $-4.5(-6.3 ;-2.7)$ \\
\hline $0-2$ & 87 & $-2.9(-3.8 ;-2.0)$ & 75 & $-4.4(-5.9 ;-2.9)$ \\
\hline$>2$ & 70 & $-3.5(-4.7 ;-2.4)$ & 59 & $-5.2(-7.1 ;-3.3)$ \\
\hline
\end{tabular}

Total number of participants is not similar for each factor as complete data are not available for all participants.

${ }^{1}$ Data are mean ( $95 \%$ confidence interval).

${ }^{2}$ Welch ANOVA was used to test significance between the groups. Statistically significant difference between the subgroups ${ }^{*}(p<0.05)^{* *}(p<0.01)^{* * *}(p<$ 0.001). Games-Howell post hoc tests for main effects. Superscript letters (a and b) indicate pairs of means that differ significantly from one another $(p<0.05)$. 


\section{Abbreviations Used}

BMI: $\quad$ Body mass index

FIN-D2D: Finnish National Diabetes Prevention

Program

GGT: $\quad$ Greater Green Triangle

GP: $\quad$ General practitioner

LSA: Lifestyle advisor

NISB: Netherlands Institute for Sport and Physical Activity

PA: Physical activity.

\section{Conflict of Interests}

No conflict of interests is declared for Bianca A. M. Schutte and Annemien Haveman-Nies. Liesbeth Preller is an employee of Netherlands Institute for Sport and Physical Activity (which owns the BeweegKuur program) and Bianca A. M. Schutte is associated with this institution. All materials relating to the intervention (and this study) are available free of charge.

\section{Acknowledgments}

The authors would like to thank all lifestyle advisors of the BeweegKuur for sending the registration files. Furthermore, we thank NISB personnel for their advice in setting up the study.

\section{References}

[1] M. M. Finucane, G. A. Stevens, M. J. Cowan et al., "National, regional, and global trends in body-mass index since 1980: systematic analysis of health examination surveys and epidemiological studies with 960 country-years and 9.1 million participants," The Lancet, vol. 377, no. 9765, pp. 557-567, 2011.

[2] X. Pi-Sunyer, "The medical risks of obesity," Postgraduate Medicine, vol. 121, no. 6, pp. 21-33, 2009.

[3] G. Whitlock, S. Lewington, P. Sherliker, R. Clarke, J. Emberson, J. Halsey et al., "Body-mass index and cause-specific mortality in 900000 adults: collaborative analyses of 57 prospective studies," The Lancet, vol. 373, no. 9669, pp. 1083-1096, 2009.

[4] F. B. Hu, M. J. Stampfer, J. E. Manson et al., "Dietary fat intake and the risk of coronary heart disease in women," The New England Journal of Medicine, vol. 337, no. 21, pp. 1491-1499, 1997.

[5] I. M. Lee, E. J. Shiroma, F. Lobelo et al., "Effect of physical inactivity on major non-communicable diseases worldwide: an analysis of burden of disease and life expectancy," The Lancet, vol. 380, no. 9838, pp. 219-229, 2012.

[6] J. Salmerón, F. B. Hu, J. E. Manson et al., "Dietary fat intake and risk of type 2 diabetes in women," American Journal of Clinical Nutrition, vol. 73, no. 6, pp. 1019-1026, 2001.

[7] G. A. Bray, A. Chatellier, C. Duncan, F. L. Greenway, E. Levy, D. H. Ryan et al., "10-year follow-up of diabetes incidence and weight loss in the Diabetes Prevention Program Outcomes Study," The Lancet, vol. 374, no. 9702, pp. 1677-1686, 2009.

[8] J. Lindström, M. Peltonen, J. G. Eriksson et al., "Improved lifestyle and decreased diabetes risk over 13 years: long-term follow-up of the randomised Finnish Diabetes Prevention Study (DPS)," Diabetologia, vol. 56, no. 2, pp. 284-293, 2013.
[9] C. Roumen, E. J. M. Feskens, E. Corpeleijn, M. Mensink, W. H. M. Saris, and E. E. Blaak, "Predictors of lifestyle intervention outcome and dropout: the SLIM study," European Journal of Clinical Nutrition, vol. 65, no. 10, pp. 1141-1147, 2011.

[10] R. E. Glasgow, E. Lichtenstein, and A. C. Marcus, "Why don't we see more translation of health promotion research to practice? Rethinking the efficacy-to-effectiveness transition," American Journal of Public Health, vol. 93, no. 8, pp. 1261-1267, 2003.

[11] P. Absetz, R. Valve, B. Oldenburg et al., "Type 2 diabetes prevention in the 'real world': one-year results of the GOAL Implementation Trial," Diabetes Care, vol. 30, no. 10, pp. 24652470, 2007.

[12] T. Laatikainen, J. A. Dunbar, A. Chapman et al., "Prevention of type 2 diabetes by lifestyle intervention in an Australian primary health care setting: Greater Green Triangle (GGT) diabetes prevention project," BMC Public Health, vol. 7, article 249, 2007.

[13] J. Lakerveld, S. D. Bot, M. J. Chinapaw et al., "Motivational interviewing and problem solving treatment to reduce type 2 diabetes and cardiovascular disease risk in real life: a randomized controlled trial," International Journal of Behavioral Nutrition and Physical Activity, vol. 10, article 47, 2013.

[14] T. Saaristo, L. Moilanen, E. Korpi-Hyövälti et al., "Lifestyle intervention for prevention of type 2 diabetes in primary health care: one-year follow-up of the finnish national diabetes prevention program (FIN-D2D)," Diabetes Care, vol. 33, no. 10, pp. 2146-2151, 2010.

[15] P. W. A. Vermunt, I. E. J. Milder, F. Wielaard, J. H. M. de Vries, H. A. M. van Oers, and G. P. Westert, "Lifestyle counseling for type 2 diabetes risk reduction in dutch primary care: results of the APHRODITE study after 0.5 and 1.5 years," Diabetes Care, vol. 34, no. 9, pp. 1919-1925, 2011.

[16] A. J. Milat, L. King, A. E. Bauman, and S. Redman, “The concept of scalability: increasing the scale and potential adoption of health promotion interventions into policy and practice," Health Promotion International, vol. 28, no. 3, pp. 285-298, 2013.

[17] J. H. M. Helmink, J. J. M. Meis, I. de Weerdt, F. N. Visser, N. K. de Vries, and S. P. J. Kremers, "Development and implementation of a lifestyle intervention to promote physical activity and healthy diet in the Dutch general practice setting: the BeweegKuur programme," International Journal of Behavioral Nutrition and Physical Activity, vol. 7, article 49, 2010.

[18] J. H. M. Helmink, S. P. J. Kremers, L. C. Van Boekel, F. N. Van Brussel-Visser, and N. K. De Vries, "Factors determining the motivation of primary health care professionals to implement and continue the 'Beweegkuur' lifestyle intervention programme," Journal of Evaluation in Clinical Practice, vol. 18, no. 3, pp. 682-688, 2012.

[19] J. H. M. Helmink, S. P. J. Kremers, L. C. van Boekel, F. N. van Brussel-visser, L. Preller, and N. K. de Vries, "The BeweegKuur programme: a qualitative study of promoting and impeding factors for successful implementation of a primary health care lifestyle intervention for overweight and obese people," Family Practice, vol. 29, no. 1, pp. i68-i74, 2012.

[20] S. Rubak, A. Sandboek, T. Lauritzen, and B. Christensen, "Motivational interviewing: a systematic review and meta-analysis," British Journal of General Practice, vol. 55, no. 513, pp. 305-312, 2005.

[21] E. Deci and R. M. Ryan, Intrinsic Motivation and SelfDetermination in Human Behaviour, Plenum, New York, NY, USA, 1985.

[22] G. C. W. Wendel-Vos, A. J. Schuit, W. H. M. Saris, and D. Kromhout, "Reproducibility and relative validity of the short 
questionnaire to assess health-enhancing physical activity," Journal of Clinical Epidemiology, vol. 56, no. 12, pp. 1163-1169, 2003.

[23] P. A. Games, H. J. Keselman, and J. C. Rogan, "Simultaneous pairwise multiple comparison procedures for means when sample sizes are unequal," Psychological Bulletin, vol. 90, no. 3, pp. 594-598, 1981.

[24] J. J. Linmans, M. G. Spigt, L. Deneer et al., "Effect of lifestyle intervention for people with diabetes or prediabetes in realworld primary care: propensity score analysis," BMC Family Practice, vol. 12, article 95, 2011.

[25] J. Lakerveld, S. D. M. Bot, M. J. Chinapaw et al., "Primary prevention of diabetes mellitus type 2 and cardiovascular diseases using a cognitive behavior program aimed at lifestyle changes in people at risk: design of a randomized controlled trial," BMC Endocrine Disorders, vol. 8, article 6, 2008.

[26] P. W. A. Vermunt, I. E. J. Milder, F. Wielaard et al., "A lifestyle intervention to reduce Type 2 diabetes risk in Dutch primary care: 2.5-year results of a randomized controlled trial," Diabetic Medicine, vol. 29, no. 8, pp. e223-e231, 2012.

[27] L. Kwak, S. P. J. Kremers, A. Walsh, and H. Brug, "How is your walking group running?” Health Education, vol. 106, no. 1, pp. 21-31, 2006.

[28] G. Duijzer, A. Haveman-Nies, S. C. Jansen, B. J. Ter, G. J. Hiddink, and E. J. Feskens, "Feasibility and potential impact of the adapted SLIM diabetes prevention intervention in a Dutch real-life setting: the SLIMMER pilot study," Patient Education and Counseling, vol. 97, no. 1, pp. 101-107, 2014.

[29] E. B. Elsman, J. N. Leerlooijer, J. Ter Beek et al., "Using the intervention mapping protocol to develop a maintenance programme for the SLIMMER diabetes prevention intervention," BMC Public Health, vol. 14, no. 1, article 1108, 2014.

[30] P. W. Vermunt, I. E. Milder, F. Wielaard et al., "Implementation of a lifestyle intervention for type 2 diabetes prevention in Dutch primary care: opportunities for intervention delivery," BMC Family Practice, vol. 13, article 79, 2012. 


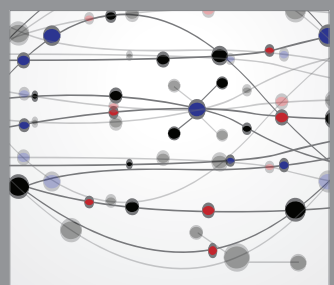

The Scientific World Journal
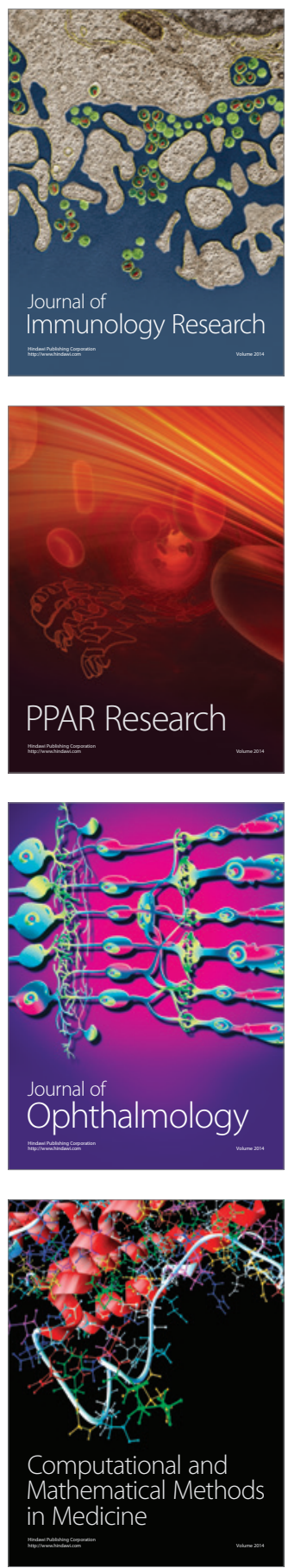

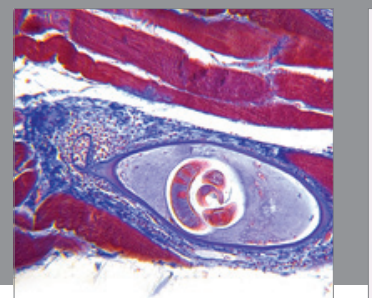

Gastroenterology

Research and Practice
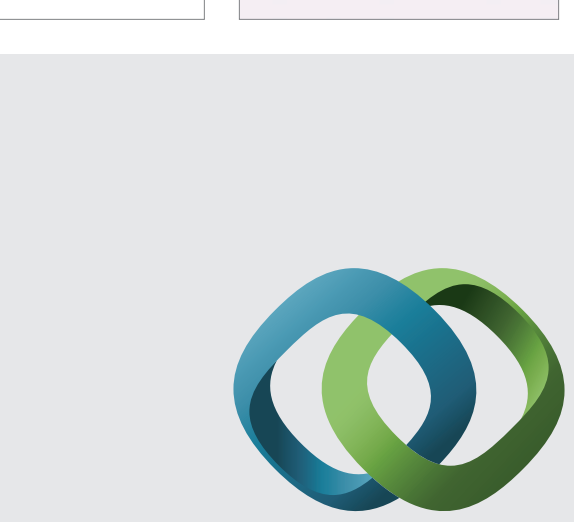

\section{Hindawi}

Submit your manuscripts at

http://www.hindawi.com
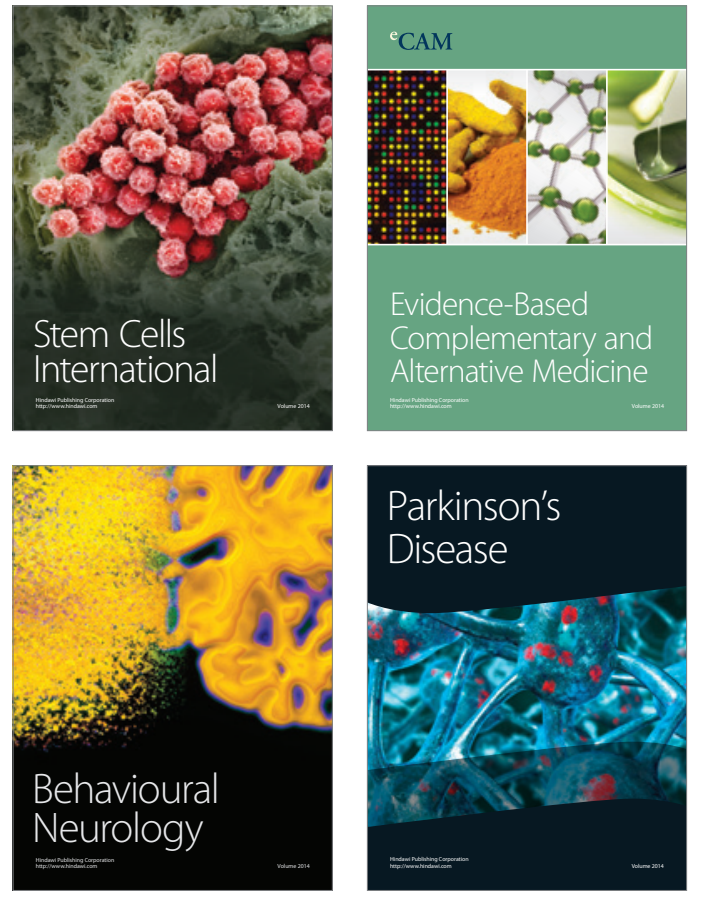
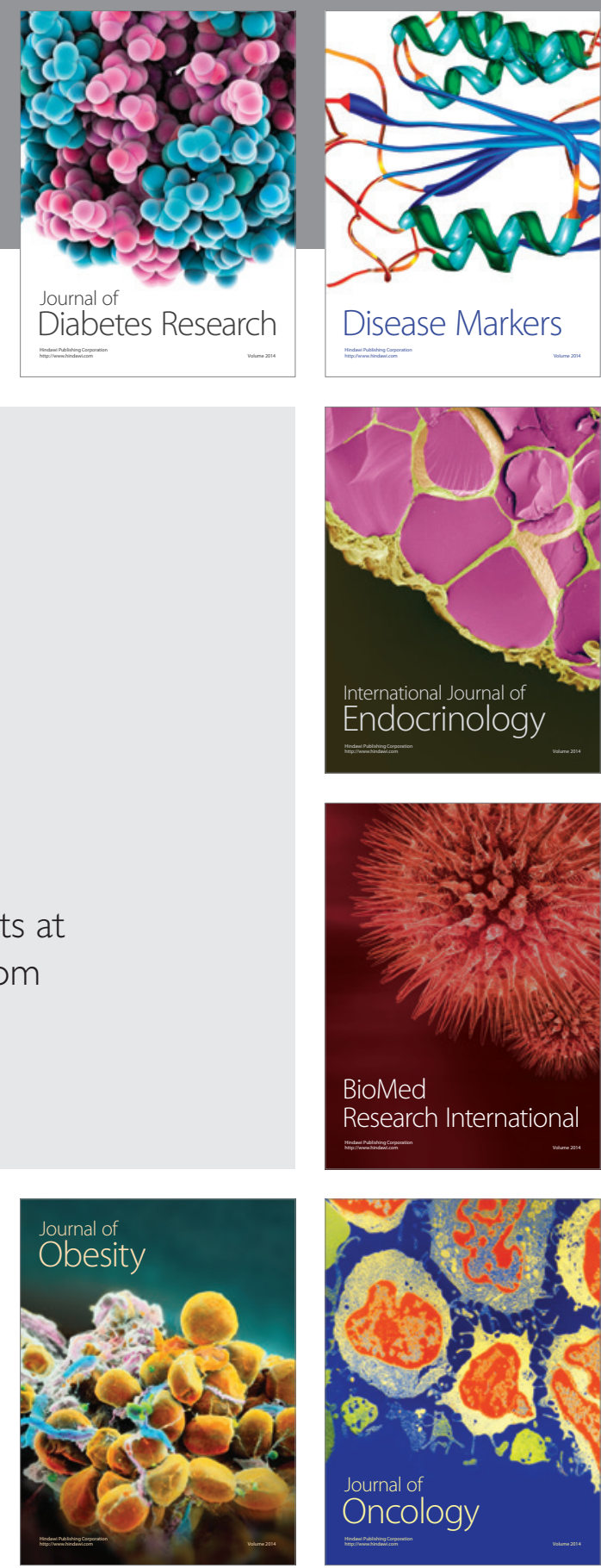

Disease Markers
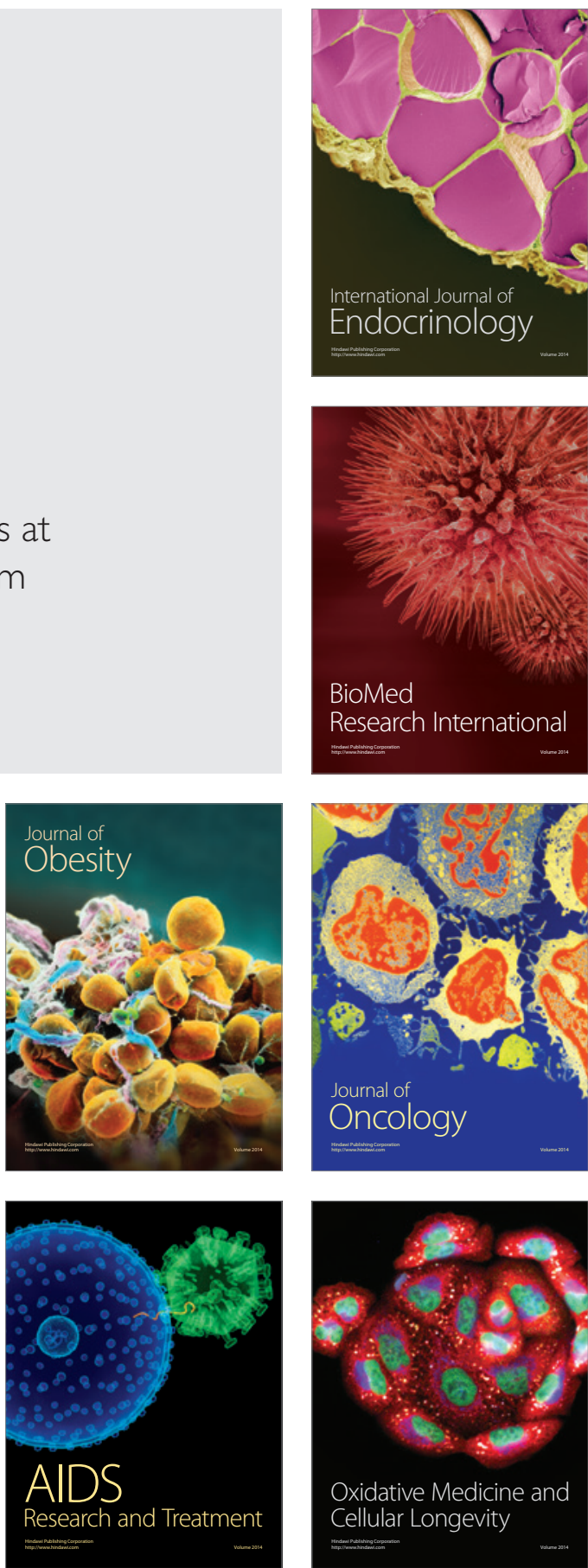